Article

\title{
The Aumann-Serrano Performance Index for Multi-Period Gambles in Stock Data
}

\author{
Jiro Hodoshima * (D) and Toshiyuki Yamawake $\mathbb{D}$ \\ Faculty of Economics, Nagoya University of Commerce and Business, 4-4 Sagamine, Komenoki-cho, Nisshin-shi, \\ Aichi 470-0193, Japan; yamawake@nucba.ac.jp \\ * Correspondence: hodoshima@nucba.ac.jp; Tel.: +81-561-73-2111; Fax: +81-561-73-1202
}

Received: 27 October 2020; Accepted: 17 November 2020; Published: 20 November 2020

\begin{abstract}
We present an empirical study of the Aumann-Serrano performance index for multi-period gambles when the underlying stochastic process is assumed to be a normal mixture process with time-varying volatility. We compare the Aumann-Serrano performance index for multi-period gambles with that for one-period gambles as well as the Sharpe ratio. Our empirical study is obtained using a selection of U.S. stock data and shows evaluation of a selection of stocks becomes more distinct in multi-period gambles than in one-period gambles in the sense that a favorable evaluation score becomes even better in multi-period gambles than in one-period gambles while an unfavorable evaluation score becomes even worse in multi-period gambles than in one-period gambles.
\end{abstract}

Keywords: Aumann-Serrano performance index; multi-period gamble; Sharpe ratio; stock data

JEL Classification: G11; C22; C46

\section{Introduction}

Providing appropriate performance measures is quite important in finance since the evaluation of assets, projects, cash flows, etc. is essential in finance via appropriate performance measures. Recently, Aumann and Serrano (2008) proposed the economic index of riskiness (hereafter the AS index) based on an axiomatic approach. While the AS index is an index of risk, it has been used in performance measures. A performance measure based on the AS index was proposed by Kadan and Liu (2014), which is obtained directly from the AS index, i.e., the reciprocal of the AS index. We use the performance measure based on the AS index proposed by Kadan and Liu (2014) to evaluate financial assets.

In this study, we intend to study multi-period gambles which stand for gambles over time where gambles stand for random variables with uncertain outcomes representing asset returns, projects, cash flows, etc. There exist many occasions where it is more appropriate to treat gambles as multi-period gambles rather than one-period gambles. For example, when we invest in financial assets, we are often concerned with long-run profits rather than short-run profits. If asset returns are observed daily and we are interested in profits five years later, then it may be more appropriate to treat daily observations as realizations of multi-period gambles rather than those of one-period gambles since we can take into account dynamic properties of returns more naturally in multi-period gambles compared to one-period gambles where returns in the future are properly discounted in multi-period gambles but not in one-period gambles. To the best of our knowledge, empirical studies of multi-period gambles seem to be rare ${ }^{1}$ although providing a theoretical framework of multi-period gambles may not be rare.

We use a setup considered by Kadan and Liu (2014) to deal with multi-period gambles based on the axiomatic approach of Aumann and Serrano (2008). In other words, we consider a T-period 
gamble $g=\left(g^{1}, g^{2}, \cdots, g^{T}\right)$ whose AS performance index $P^{A S(g)}$ is given by the unique solution of the implicit equation

$$
\sum_{t=1}^{T} \rho^{t-1} E\left[\exp \left(-P^{A S}(g) \cdot g^{t}\right)\right]=\sum_{t=1}^{T} \rho^{t-1},
$$

where $\rho \in(0,1)$ denotes a discount factor. The above equation is equal to Equation (8) of Kadan and Liu (2014). To the best of our knowledge no studies of the above AS performance index $P^{A S}(g)$ for multi-period gambles were conducted since it was proposed by Kadan and Liu (2014). We shall make an empirical study of the above AS performance index using a selection of U.S. stock market data. We consider as multi-period gambles a stochastic process given by a normal mixture process with time-varying volatility. Please note that one must assume some models of the dynamic process of $g$ since the implicit equation given above involves expectation of $g^{t}$ at each time $t$ in order to obtain the AS performance index $P^{A S(\boldsymbol{g})}$ for multi-period gambles. We remark that a normal mixture process is a process with its distribution each time being independently and identically distributed (i.i.d.). A normal mixture process is a simple stochastic process but is known to capture well characteristics of distributions often observed in financial data such as skewed and heavy tail distributions as well as symmetric and multi-modal distributions.

We incorporate time-varying volatility into a normal mixture process in this study. Time-varying volatility is another stylized feature often observed in financial data. We use a family of generalized autoregressive conditional heteroskedastic (GARCH) models proposed by Bollerslev (1986), which is a generalization of the autoregressive conditional heteroskedastic (ARCH) model originally proposed by Engle (1982), as a time-varying volatility model. By combining a normal mixture process with time-varying volatility, we can create state-dependent volatility models which allow different volatility responses to stock price shocks in stable and stressful market conditions, which is not possible under single-state GARCH models allowing only one mechanism of volatility to market shocks. State-dependent volatility models are more flexible to capture characteristics of financial data than single-state volatility models. When we use a family of GARCH models for a selection of U.S. stock market data, we try to take into account the so-called leverage effect of negative returns with more influence on the underlying volatility than positive returns. When we evaluate multi-period gambles, we should capture well the underlying process of multi-period gambles to be used in the performance measure. Otherwise we cannot obtain the relevant evaluation. Therefore, modeling appropriately the underlying stochastic process is crucial to conduct evaluation based on the performance measure for multi-period gambles. Since providing proper evaluation for financial assets, projects, cash flows, etc. is essential to investors, financial managers, and regulators, our approach should be very useful to them.

We compare performance of a selection of U.S. stocks when they are evaluated as realizations of multi-period gambles with that when they are evaluated as realizations of one-period gambles. Performance of one-period gambles is computed by the AS performance index for one-period gambles, i.e., the solution $P^{A S}(g)$ of the implicit equation

$$
E\left[\exp \left(-P^{A S}(g) g\right)\right]=1
$$

for one-period gambles $g$, assuming the underlying stochastic process of gambles $g$ to be a normal mixture process with time-invariant volatility, a special case of the normal mixture process with time-varying volatility of GARCH families, i.e., the assumption of the stochastic process of multi-period gambles in this study. The difference of the assumption of the underlying stochastic process of multi-period gambles from that of one-period gambles is whether the volatility process is time-varying or time-invariant. Therefore, we explicitly model time-varying volatility as GARCH families in evaluation of multi-period gambles with late return observations properly discounted. On the other hand, we assume volatility to be time-invariant in evaluation of one-period gambles with late return observations not discounted. We will examine the consequences of the difference in the model of 
multi-period gambles from that of one-period gambles in this study. The data we use in this study covered the global financial crisis where tremendous volatility clustering was observed in stock markets. Therefore, we anticipate in our sample period the AS performance index for multi-period gambles is more relevant than that for one-period gambles since the former takes into account volatility clustering but the latter does not. In addition, our anticipation is verified by our empirical results. Our results show when stocks are more poorly evaluated by the AS performance index for one-period gambles than the Sharpe ratio, then they are evaluated even more poorly in multi-period gambles than in one-period gambles. On the other hand, when stocks are more favorably evaluated by the AS performance index for one-period gambles than the Sharpe ratio, then they are evaluated more favorably in multi-period gambles than in one-period gambles. In other words, evaluation of a selection of stocks becomes more distinct in multi-period gambles than in one-period gambles in the sense that a favorable evaluation score becomes even better in multi-period gambles than in one-period gambles while an unfavorable evaluation score becomes even worse in multi-period gambles than in one-period gambles.

The rest of the paper is organized as follows. In Section 2, we present the literature review related to our method. In Section 3, we present our setup and the assumptions of the underlying stochastic process. In Section 4, we show our empirical study using a selection of U.S. stock data. In Section 5 , we present concluding comments.

\section{Literature Review}

In this section, we provide the literature review related to our study. The AS index has received a lot of attention in finance (see, e.g., Foster and Hart 2009; Hart 2011; Homm and Pigorsch 2012a; Kadan and Liu 2014; Sculze 2014; Niu et al. 2018; Hodoshima and Miyahara 2020). Another performance measure based on the AS index in the literature is the economic performance measure proposed by Homm and Pigorsch (2012a) for one-period gambles, which is a modification of the Sharpe ratio by replacing standard deviation by the AS index. Hodoshima (2020) compared the economic performance measure proposed by Homm and Pigorsch (2012a) and the AS performance index for one-period gambles proposed by Kadan and Liu (2014) and showed the latter is superior to the former.

There also exist plenty of other performance measures. See, e.g., Cogneau and Hubner (2009a, 2009b), and Cherny and Madan (2009). While these performance measures were compared by many studies, these preceding studies were mainly analyzed as static performance measures, i.e., the underlying gambles are assumed to be one-period gambles. Also many of these performance measures are proposed in ad hoc ways. On the other hand, the performance measure based on the AS index is based on an axiomatic approach. Although performance measures based on the AS index have been used to evaluate performance of various funds, portfolios, individual assets, etc. (see, e.g., Homm and Pigorsch 2012a; Kadan and Liu 2014; Hodoshima 2019; Hodoshima and Otsuki 2019), they are confined to only one-period gambles. Therefore, our use of the AS performance index for multi-period gambles in empirical studies is the first trial in the literature.

A normal mixture process or a normal mixture distribution has been studied in the statistics and econometrics literature for several decades. See, e.g., Everitt and Hand (1981); McLachlan and Peel (2000); and Titterington et al. (1985). There are also many applications of the normal mixture process in finance (cf., e.g., Kon 1984; Alexander 2004; Hodoshima 2019). To capture the leverage effect, we employ the asymmetric GARCH model (hereafter AGARCH model) considered by Engle (1990) and Engle and Ng (1993) and the model proposed by Glosten et al. (1993) (hereafter GJR model). Therefore, we use a normal mixture process with time-varying GARCH volatility taking into account the leverage effect as the underlying stochastic process for multi-period gambles. Models we consider in this paper are similar to those treated in Alexander and Lazar (2009). However, we use an estimation method different from Alexander and Lazar (2009) who used the maximum likelihood estimator (MLE). In other words, we use an empirical characteristic function approach due to $\mathrm{Xu}$ and Wirjanto (2010) as an estimation method for our models. In particular, we estimate a normal 
mixture process with components up to three, which is different from the previous empirical study of Alexander and Lazar (2009), where components in the normal mixture process were restricted to two. It was stated in Alexander and Lazar (2009) that in estimating a normal mixture process with three components by the MLE, convergence was harder to attain. On the other hand, we do not encounter such problems in our empirical study by an empirical characteristic function approach.

\section{The Model for Multi-Period Gambles}

In this section, we present the theoretical framework for multi-period gambles and the normal mixture process with time-varying GARCH volatility as the process of multi-period gambles.

As shown by Kadan and Liu (2014), our theoretical framework for multi-period gambles is given as follows. We consider a T-period gamble $g=\left(g^{1}, g^{2}, \ldots, g^{T}\right)$ where each $g^{t}$ denotes a gamble at time $t(t=1,2, \cdots, T)$. We consider an investor with a time-separable utility function $U: R^{T} \longmapsto R$ with the form given by

$$
U\left(w^{1}, w^{2}, \cdots, w^{T}\right)=\sum_{t=1}^{T} \rho^{t-1} u\left(w^{t}\right)
$$

where $u$ denotes a utility function, $\rho \in(0,1)$ is a discount factor, and $\left(w^{1}, w^{2}, \cdots, w^{T}\right)$ denotes the level of wealth from 1 to T. Notice domain of the level of wealth is not, unlike Kadan and Liu (2014), restricted to positive region since the AS performance index can be defined in positive region as well as negative region (cf. Hodoshima and Miyahara 2020). First we present two definitions to provide ordering of one-period gambles.

Definition 1 (Definition 2 of Kadan and Liu (2014)). A gamble $g$ is wealth-uniformly rejected by an investor with utility function $u$, if $u$ rejects $g$ at all initial wealth levels $w_{0}$.

Definition 2 (Definition 3 of Kadan and Liu (2014)). A gamble $g$ wealth-uniformly dominates a gamble $\boldsymbol{g}^{\prime}$ if whenever $g$ is wealth-uniformly rejected by a utility function $u, g^{\prime}$ is also wealth-uniformly rejected by $u$.

Then, the following proposition provides a property of the AS performance index for one-period gambles.

Proposition 1 (Proposition 1 of Kadan and Liu (2014)). Wealth-uniform dominance induces a complete order on the set $\mathcal{G}$ of one-period gambles. This order can be represented by a performance index $P^{A S}(g)$ assigned to any gamble $g \in \mathcal{G}$, which is given by the unique solution to the implicit equation

$$
E\left[\exp \left(-P^{A S}(g) g\right)\right]=1 .
$$

That is, for any two gambles $g$ and $g^{\prime}, g$ wealth-uniformly dominates $g^{\prime}$ if and only if $P^{A S}(g) \geq P^{A S}\left(g^{\prime}\right)$.

The property of the above proposition is extended to multi-period gambles as follows.

Proposition 2 (Proposition 3 of Kadan and Liu (2014)). Wealth-uniform dominance induces a complete order on $\mathcal{G}^{T}$. This order can be represented by a performance index $P^{A S}(g)$ assigned to any T-period gamble $g=\left(g^{1}, g^{2}, \cdots, g^{T}\right) \in \mathcal{G}^{T}$, which is given by the unique solution to the implicit equation

$$
\sum_{t=1}^{T} \rho^{t-1} E\left[\exp \left(-P^{A S}(g) \cdot g^{t}\right)\right]=\sum_{t=1}^{T} \rho^{t-1}
$$

That is, for any two gambles $g$ and $\boldsymbol{g}^{\prime}, \boldsymbol{g}$ wealth-uniformly dominates $\boldsymbol{g}^{\prime}$ if and only if $P^{A S}(g) \geq P^{A S}\left(\boldsymbol{g}^{\prime}\right)$.

There have been no empirical studies of multi-period gambles yet so that we do not know what kind of consequences the concept of multi-period gambles entails. In this study, we use a selection of 
U.S. stocks as examples of multi-period gambles to compute the AS performance index given by the solution of the implicit Equation (3). We also use the same data as examples of one-period gambles to compare the AS performance index, given by the solution of the implicit Equation (2), with the AS performance index for multi-period gambles, given by the solution of the implicit Equation (3). Furthermore, we compare the two different AS performance indexes with the Sharpe ratio computed from data.

To derive the AS performance index for multi-period gambles, we employ several parametric models under the maintained assumption that the underlying stochastic process of multi-period gambles follows a normal mixture process with time-varying volatility of GARCH families. We follow Alexander and Lazar (2009); Haas et al. (2004); and Xu and Wirjanto (2010) to assume that the return $\boldsymbol{X}_{t}$ of an asset is given by

$$
\boldsymbol{X}_{t}=\epsilon_{t}
$$

where $\epsilon_{t}$ follows a mixture of $K$ normal distributions with a time-varying volatility process

$$
\epsilon_{t} \mid I_{t-1} \sim \pi_{k} N\left(\mu_{k}, \sigma_{k, t}^{2}\right)
$$

for $t=1, \cdots, T$ and $k=1, \cdots, K$, where $N\left(\mu_{k}, \sigma_{k, t}^{2}\right)$ denotes normal distribution with mean $\mu_{k}$ and variance $\sigma_{k, t}^{2}, I_{t-1}$ is the information set up to time $t-1,0 \leq \pi_{k} \leq 1$, and $\sum_{k=1}^{K} \pi_{k}=1$. We assume that the conditional variance of the $k$-th component follows three possible processes;

(1) GARCH(1,1) process

$$
\sigma_{k, t}^{2}=\omega_{k}+\alpha_{k} \epsilon_{t-1}^{2}+\beta_{k} \sigma_{k, t-1}^{2}
$$

(2) Asymmetric GARCH(1,1) $(\mathrm{AGARCH}(1,1))$ process

$$
\sigma_{k, t}^{2}=\omega_{k}+\alpha_{k}\left(\epsilon_{t-1}-\lambda_{k}\right)^{2}+\beta_{k} \sigma_{k, t-1}^{2}
$$

(3) GJR(1,1) process (the model based on Glosten et al. (1993))

$$
\sigma_{k, t}^{2}=\omega_{k}+\alpha_{k} \epsilon_{t-1}^{2}+\lambda_{k} d_{t-1}^{-} \epsilon_{t-1}^{2}+\beta_{k} \sigma_{k, t-1}^{2}
$$

where $d_{t}^{-}=1$ if $\epsilon_{t}<0$ and 0 otherwise, component conditional variances depend on the previous innovation $\epsilon_{t-1}$ as well as their own previous conditional variances.

We make component conditional variances not dependent on the previous conditional variances of other components. Then, the conditional mean, variance, skewness, and kurtosis of $X_{t}$ given the information set up to time $t-1$ are given respectively by

$$
\begin{aligned}
\mu & =\sum_{k=1}^{K} \pi_{k} \mu_{k} \\
\sigma_{t}^{2} & =\sum_{k=1}^{K} \pi_{k}\left(\sigma_{k, t}^{2}+\mu_{k}^{2}\right)-\mu^{2} \\
\tau_{t} & =\frac{1}{\sigma_{t}^{3}} \sum_{k=1}^{K} \pi_{k}\left(\mu_{k}-\mu\right)\left[3 \sigma_{k, t}^{2}+\left(\mu_{k}-\mu\right)^{2}\right] \\
\kappa_{t} & =\frac{1}{\sigma_{t}^{4}} \sum_{k=1}^{K} \pi_{k}\left[3 \sigma_{k, t}^{4}+6\left(\mu_{k}-\mu\right)^{2} \sigma_{k, t}^{2}+\left(\mu_{k}-\mu\right)^{4}\right] .
\end{aligned}
$$

When $X_{t}$ follows the above normal mixture process with time-varying volatility of GARCH families, the following equality holds for $E\left[\exp \left(-P^{A S}(g) \cdot g^{t}\right)\right]$ in the implicit Equation (3) of the AS performance index:

$$
E\left[\exp \left(-P^{A S}(g) \cdot g^{t}\right)\right]=\sum_{k=1}^{K} \pi_{k} \exp \left(-\mu_{k} P^{A S}(g)+\sigma_{k, t}^{2} P^{A S}(g)^{2} / 2\right)
$$


since the moment-generating function (MGF) $E[\exp (s \boldsymbol{Y})]$ of a random variable $\boldsymbol{Y}$ is given by

$$
\exp \left(\mu s+\sigma^{2} s^{2} / 2\right)
$$

when $Y$ follows normal distribution $N\left(\mu, \sigma^{2}\right)$. Notice $E\left[\exp \left(-P^{A S}(g) \cdot g^{t}\right)\right]$ is, besides the minus sign, nothing but the MGF of $g^{t}$ as a function of $P^{A S}(g)$.

When a multi-period gamble $g=\left(g^{1}, g^{2}, \cdots, g^{T}\right)$ follows the normal mixture process with the above GARCH families, the AS performance index for the gamble $g=\left(g^{1}, g^{2}, \ldots, g^{T}\right)$ is given by the unique solution $P^{A S}(g)$ to the implicit equation

$$
\sum_{t=1}^{T} \rho^{t-1} \sum_{k=1}^{K} \pi_{k} \exp \left(-\mu_{k} P^{A S}(g)+\sigma_{k, t}^{2} P^{A S}(g)^{2} / 2\right)=\sum_{t=1}^{T} \rho^{t-1}
$$

where $\rho \in(0,1)$ is a discount factor.

In this study, we seek to obtain the AS performance index for multi-period gambles empirically. Providing a sufficient condition for existence of the AS performance index for multi-period gambles as in that for one-period gambles given by Aumann and Serrano (2008); Homm and Pigorsch (2012b); and Sculze (2014) is beyond the scope of this study.

To obtain the AS performance index for multi-period gambles, we first estimate the parametric models for the underlying stochastic model of the normal mixture process with time-varying volatility of GARCH families. To estimate the parametric models, we use an empirical characteristic function (ECF) approach of $\mathrm{Xu}$ and Wirjanto (2010) which has several advantages as a method of estimating the parametric models: a closed-form objective distance function is available, the estimator has strong consistency and asymptotic normality, and the characteristic function is always uniformly bounded, unlike the likelihood function which is not always bounded over its parameter space (cf. Xu and Wirjanto 2010).

We employ the continuous empirical characteristic function (CECF) approach by $\mathrm{Xu}$ and Wirjanto (2010) to estimate the AS performance index when the underlying stochastic process is given by the normal mixture process with time-varying volatility of GARCH families. The CF associated with Equations (4) and (5) is defined by

$$
C_{t}(r, \theta)=E\left[e^{i r X_{t}}\right]=\sum_{k=1}^{K} \pi_{k} \exp \left(i \mu_{k} r-\frac{1}{2} \sigma_{k, t}^{2} r^{2}\right)
$$

where $i=\sqrt{-1}$ and $\theta$ denotes the set of parameters in the model.

The ECF of the above equation is given by

$$
C_{t}\left(r, X_{t}\right)=\exp \left(i r X_{t}\right)
$$

Then we consider the following distance measure given by

$$
D_{t}\left(\theta ; \boldsymbol{X}_{t}\right)=\int\left|C_{t}\left(r, \boldsymbol{X}_{t}\right)-C_{t}(r, \theta)\right|^{2} \exp \left(-b r^{2}\right) d r .
$$

We have the following result for the closed-form expression of the above distance function $D_{t}\left(\theta ; \boldsymbol{X}_{t}\right)$. 
Proposition 3 (Proposition 1 of $\mathrm{Xu}$ and Wirjanto (2010)). If the return $\boldsymbol{X}_{t}$ is generated from Equations (4) and (5) and the distance measure under the CECF is given by Equation (15), then the closed-form-expression for the distance measure $D_{t}\left(\theta ; \boldsymbol{X}_{t}\right)$ is given by

$$
\begin{aligned}
D_{t}\left(\theta ; \boldsymbol{X}_{t}\right)= & \sqrt{\frac{\pi}{b}}+\sum_{k=1}^{K} \pi_{k}^{2} \sqrt{\frac{\pi}{b+\sigma_{k, t}^{2}}} \\
& -2 \sum_{k=1}^{K}\left(\pi_{k} \sqrt{\frac{\pi}{\frac{1}{2} \sigma_{k, t}^{2}+b}} \exp \left(-\frac{\left(\boldsymbol{X}_{t}-\mu_{k}\right)^{2}}{4 b+2 \sigma_{k, t}^{2}}\right)\right) \\
& +2 \sum_{k \neq h} \pi_{k} \pi_{h} \sqrt{\frac{\pi}{b+\frac{1}{2}\left(\sigma_{k, t}^{2}+\sigma_{h, t}^{2}\right)}} \\
& \times \exp \left(-\frac{\left(\mu_{k}-\mu_{h}\right)^{2}}{4 b+2\left(\sigma_{k, t}^{2}+\sigma_{h, t}^{2}\right)}\right) .
\end{aligned}
$$

We remark the conditional variance $\sigma_{k, t}^{2}$ of the k-th component in the closed-form-expression given above can be any of the three possible processes of GARCH families given above. In other words, the closed-form-expression (16), originally for the standard GARCH models, continues to hold for other forms of GARCH families such as AGARCH and GJR models. We employ $b=1$ when we implement estimation by minimizing the closed-form expression as in $X u$ and Wirjanto (2010).

The CECF estimation of the model is to minimize $D(\theta)=\sum_{t=1}^{T} D_{t}\left(\theta ; \boldsymbol{X}_{t}\right)$ with respect to the set of unknown parameters in the model. The following result holds for the asymptotic normality result.

\section{Proposition 4.}

$$
\sqrt{T}(\hat{\theta}-\theta) \Longrightarrow N\left(0, \Lambda^{-1} \Omega \Lambda^{-1}\right)
$$

where $\hat{\theta}$ denotes the estimator by the CECF approach, $\Longrightarrow$ denotes convergence in distribution, $\Lambda=E\left[\frac{\partial^{2} D(\theta)}{\partial \theta \partial \theta^{\prime}}\right]$, and $\Omega=E\left[\frac{\partial D(\theta)}{\partial \theta} \frac{\partial D(\theta)}{\partial \theta^{\prime}}\right]$.

See Heathcote (1977) for the proof of the above proposition.

\section{The Empirical Estimation Results}

In this section, we estimate the AS performance index for multi-period gambles by the CECF approach described in the previous section when the underlying stochastic process is assumed to be the normal mixture process with time-varying volatility of GARCH families. We employ a selection of U.S. stock return data to estimate the AS performance index. We estimate the AS performance index for multi-period gambles as well as for one-period gambles and compare the two AS performance indexes to find out the consequences of the assumption for multi-period gambles. The AS performance index for one-period gambles is obtained as a parametric MLE assuming the underlying stochastic process follows the normal mixture process with time-invariant volatility, which is a special case of the normal mixture process with time-varying volatility of GARCH families, i.e., the assumption of the underlying stochastic process for the AS performance index for multi-period gambles. We use the two market indexes of the Dow Jones Industrial Average (DOW) and Nasdaq Composite Index (NASDAQ) and individual stocks of Johnson and Johnson (JNJ), Amazon, and Microsoft as a selection of U.S. stocks. We employ daily return data of these stocks from January 2, 2008, till April 28, 2017. We show the Sharpe ratio for these data in addition to the AS performance index for multi-period gambles as well as for one-period gambles. The Sharpe ratio is computed directly from data. The risk-free rate is obtained from the Treasury bill rate data, downloaded from Ken French's homepage.

We first provide summary statistics for the stock return data in Table 1. Summary statistics are mean, standard deviation (s.d.), skewness, and kurtosis. Mean ranges from 0.027 in DOW to 0.129 in 
Amazon. Standard deviation ranges from 1.054 in Johnson \& Johnson to 2.485 in Amazon. Therefore, Amazon is a stock with the highest mean and the highest risk. All the stocks are positively skewed with the exception of NASDAQ. All the stocks have heavy tails compared to the normal distribution.

Table 1. Summary Statistics of the Stock Returns. Table 1 presents summary statistics of the stock return data we study in this paper, i.e., mean, standard deviation (s.d.), skewness, and kurtosis.

\begin{tabular}{ccccc}
\hline Name & Mean & s.d. & Skewness & Kurtosis \\
\hline DOW & 0.027 & 1.221 & 0.157 & 13.836 \\
NASDAQ & 0.045 & 1.407 & -0.071 & 10.451 \\
JNJ & 0.044 & 1.054 & 0.690 & 16.582 \\
Amazon & 0.129 & 2.485 & 0.977 & 14.435 \\
Microsoft & 0.044 & 1.793 & 0.467 & 13.784 \\
\hline
\end{tabular}

JNJ stands for Johnson \& Johnson.

We follow Engle and $\mathrm{Ng}$ (1993) to test serial correlation in levels and squares of stock return data and to examine if the value of $\epsilon_{t-1}$ influences current volatility. Table 2 provides results of Ljung-Box statisics $^{2}$ of serial correlation of the twelfth-order for levels and squares of stock return data as well as results of the sign bias test statistic, negative size bias test statistic, positive size bias test statistic, and joint test statistic described in Engle and Ng (1993). The sign bias test statistic, negative size bias test statistic, and positive sign bias test statistic given in Engle and $\mathrm{Ng}$ (1993) are respectively the t-test statistic of the explanatory variable $d_{t-1}^{-}, d_{t-1}^{-} \epsilon_{t-1}$, and $d_{t-1}^{+} \epsilon_{t-1}$ in the regression equation of the normalized residual as the dependent variable where $d_{t-1}^{-}=1$ if $\epsilon_{t-1}<0$ and 0 otherwise, $d_{t-1}^{+}=d_{t-1}^{-}+1$, and the normalized residual is the residual divided by the conditional standard deviation estimate. The joint test statistic is the Lagrange multiplier (LM) test statistic for adding the three variables of the sign bias, negative size bias, and positive sign bias in the regression equation of the normalized residual as the dependent variable. Table 2 shows all the stocks are serially uncorrelated in levels but serially highly correlated in squares of stock returns, which are conformable with the stylized facts of financial data. With respect to the effects of the value of $\epsilon_{t-1}$ on current volatility, the negative size bias test is always highly significant, which is the same as in Engle and $\mathrm{Ng}$ (1993) for the Japanese stock index data in their study. The sign bias test statistic is positive and highly significant for the two indexes but insignificant for two individual stocks of Amazon and Microsoft. Later, we will see the best model in Amazon and Microsoft are GARCH $(1,1)$ three components models, the most complicated models, where the effect of the negative sign of $\epsilon_{t-1}$ being not in simple forms may be due to this insignificant result. The positive size bias test is not significant for the two indexes, which is the same as in Engle and Ng (1993) for the Japanese stock index in their study. However, it is positive and significant for the three individual stocks. Therefore, the negative size bias test is significant while the positive size bias test is not significant in the two indexes of the DOW and NASDAQ, indicating the effect of $\epsilon_{t-1}$ on current volatility is asymmetric in the DOW and NASDAQ. On the other hand, the negative size bias test and the positive size bias test are both significant in Johnson \& Johnson, Amazon, and Microsoft, indicating the effect of $\epsilon_{t-1}$ on current volatility is symmetric in the three individual stocks. The joint test is highly significant for all the stocks.

2 The Ljung-Box statistics we use here are modified ones by Diebold (1988) who corrected the original Ljung-Box statistic which is known to reject the null hypothesis too often. 
Table 2. Diagnostic Test Results for the Stock Returns.

\begin{tabular}{cccccc}
\hline Name & DOW & NASDAQ & JNJ & Amazon & Microsoft \\
\hline Ljung-Box(12) in levels & 14.613 & 8.413 & 17.048 & 8.733 & 17.016 \\
Ljung-Box(12) in squares & $139.398^{* *}$ & $166.417^{* *}$ & $94.072^{* *}$ & $103.016^{* *}$ & $93.730^{* *}$ \\
Sign Bias & $3.072^{* *}$ & $3.302^{* *}$ & $2.259^{*}$ & -0.455 & 1.490 \\
Negative Size Bias & $-12.016^{* *}$ & $-11.047^{* *}$ & $-11.474^{* *}$ & $-4.738^{* *}$ & $-7.275^{* *}$ \\
Positive Size Bias & $1.952^{* *}$ & 1.770 & $2.610^{* *}$ & $2.449^{*}$ & $3.423^{* *}$ \\
Joint Test & $164.971^{* *}$ & $142.924^{* *}$ & $167.640^{* *}$ & $43.036^{* *}$ & $84.583^{* *}$ \\
\hline
\end{tabular}

JNJ stands for Johnson \& Johnson. One and two asterisks of * and ${ }^{* *}$ indicate significance at the 5 and 1 percent levels respectively.

Table 2 provides results of Ljung-Box statistics, modified by Diebold (1988), of serial correlation of the twelfth-order in levels and squares of the stock return data as well as results of the sign bias test statistic, negative size bias test statistic, positive size bias test statistic, and joint test statistic.

We then show the best model for each stock with respect to the Bayesian Information Criterion (BIC). We show the best estimation results for three classes of $\operatorname{GARCH}(1,1)$ families, i.e., $\operatorname{GARCH}(1,1)$ model, AGARCH $(1,1)$ model, and GJR $(1,1)$ model in Tables 3-7. Standard errors are obtained using asymptotic variance given in Equation (17) in Proposition 4. In models with more than one component, probability of one component is determined automatically by the restriction of the sum of probabilities of all the components being equal to one so that its standard error is not given. The best estimation results are the AGARCH(1,1) two components model for the two indexes and the GARCH $(1,1)$ two and three components models for the three individual stocks. The AGARCH $(1,1)$ two components model as the best model in the DOW and NASDAQ conform to the results of the negative size bias test and positive size bias test described above. The $\mathrm{GARCH}(1,1)$ two and three components models as the best model in the three individual stocks are also in harmony with the results of the negative size bias test and positive size bias test explained above, indicating the effect of $\epsilon_{t-1}$ on current volatility is symmetric in the three individual stocks.

We describe the estimation results only for the best model in each stock below although the best estimation results for the three classes of $\operatorname{GARCH}(1,1)$ families are provided in Tables 3-7. The best model in the stock indexes DOW and NASDAQ are both AGARCH $(1,1)$ two components models. They are composed of a dominant state with negative mean and a positive shock state with positive mean. The estimate of $\alpha$, the effect of previous innovation $\epsilon_{t-1}$, is larger in the dominant state with negative mean than in the positive shock state with positive mean, indicating the effect of previous innovation is larger in the dominant state than in the positive shock state. The estimate of $\beta$, the effect of previous conditional variance $\sigma_{k, t-1}^{2}$, is smaller in the dominant state with negative mean than in the positive shock state with positive mean, indicating the effect of previous conditional variance is short-lived in the dominant state than in the positive shock state. The estimate of $\lambda$ is larger in the dominant state than in the positive shock state, indicating the effect of previous innovation of minus sign on volatility is larger in the dominant state than in the positive shock state.

Table 3 shows the best estimation results for the DOW in the three classes of $\operatorname{GARCH}(1,1)$ families, i.e., GARCH $(1,1)$ model, AGARCH $(1,1)$ model, and GJR(1,1) model. Standard errors, shown in parentheses, are obtained using asymptotic variance given in Equation (17) in Proposition 4. In models with more than one component, probability of one component is determined automatically by the restriction of the sum of probabilities of all the components being equal to one so that its standard error is not given. 
Table 3. Estimation of the Three Classes of GARCH(1,1) Families in the DOW.

\begin{tabular}{|c|c|c|c|c|c|c|}
\hline \multicolumn{7}{|c|}{ GARCH-2component } \\
\hline$\mu_{1}$ & $\mu_{2}$ & $\omega_{1}$ & $\alpha_{1}$ & $\beta_{1}$ & $\omega_{2}$ & $\alpha_{2}$ \\
\hline $\begin{array}{c}0.162 \\
(0.030)\end{array}$ & $\begin{array}{c}-0.443 \\
(0.271)\end{array}$ & $\begin{array}{c}0.002 \\
(0.004)\end{array}$ & $\begin{array}{c}0.059 \\
(0.008)\end{array}$ & $\begin{array}{c}0.903 \\
(0.008)\end{array}$ & $\begin{array}{c}0.000 \\
(0.080)\end{array}$ & $\begin{array}{c}0.366 \\
(0.043)\end{array}$ \\
\hline$\beta_{2}$ & $\pi_{1}$ & $\pi_{2}$ & $\mathrm{BIC}$ & & & \\
\hline $\begin{array}{c}0.831 \\
(0.012)\end{array}$ & $\begin{array}{c}0.755 \\
(0.073)\end{array}$ & 0.245 & 6289.892 & & & \\
\hline \multicolumn{7}{|c|}{ GJR-1component } \\
\hline$\mu_{1}$ & $\omega_{1}$ & $\alpha_{1}$ & $\lambda_{1}$ & $\beta_{1}$ & BIC & \\
\hline $\begin{array}{c}0.048 \\
(0.001)\end{array}$ & $\begin{array}{c}0.014 \\
(0.001)\end{array}$ & $\begin{array}{c}0.000 \\
(0.029)\end{array}$ & $\begin{array}{c}0.175 \\
(0.013)\end{array}$ & $\begin{array}{c}0.896 \\
(0.023)\end{array}$ & 6193.376 & \\
\hline \multicolumn{7}{|c|}{ AGARCH-2component } \\
\hline$\mu_{1}$ & $\mu_{2}$ & $\omega_{1}$ & $\alpha_{1}$ & $\lambda_{1}$ & $\beta_{1}$ & $\omega_{2}$ \\
\hline $\begin{array}{l}-0.054 \\
(0.129)\end{array}$ & $\begin{array}{c}0.179 \\
(0.192)\end{array}$ & $\begin{array}{c}0.000 \\
(0.011)\end{array}$ & $\begin{array}{c}0.169 \\
(0.103)\end{array}$ & $\begin{array}{c}0.591 \\
(0.115)\end{array}$ & $\begin{array}{c}0.829 \\
(0.035)\end{array}$ & $\begin{array}{c}0.000 \\
(0.022) \\
\end{array}$ \\
\hline$\alpha_{2}$ & $\lambda_{2}$ & $\beta_{2}$ & $\pi_{1}$ & $\pi_{2}$ & $\mathrm{BIC}$ & \\
\hline $\begin{array}{c}0.030 \\
(0.102)\end{array}$ & $\begin{array}{c}0.370 \\
(0.425)\end{array}$ & $\begin{array}{c}0.936 \\
(0.193)\end{array}$ & $\begin{array}{c}0.612 \\
(0.678)\end{array}$ & 0.388 & 6182.353 & \\
\hline
\end{tabular}

Table 4 shows the best estimation results for the NASDAQ in the three classes of GARCH $(1,1)$ families, i.e., GARCH $(1,1)$ model, AGARCH $(1,1)$ model, and GJR $(1,1)$ model. Standard errors, shown in parentheses, are obtained using asymptotic variance given in Equation (17) in Proposition 4. In models with more than one component, probability of one component is determined automatically by the restriction of the sum of probabilities of all the components being equal to one so that its standard error is not given.

Table 4. Estimation of the Three Classes of GARCH(1,1) Families in the NASDAQ.

\begin{tabular}{|c|c|c|c|c|c|c|}
\hline \multicolumn{7}{|c|}{ GARCH-2component } \\
\hline$\mu_{1}$ & $\mu_{2}$ & $\omega_{1}$ & $\alpha_{1}$ & $\beta_{1}$ & $\omega_{2}$ & $\alpha_{2}$ \\
\hline $\begin{array}{c}-0.186 \\
(0.007)\end{array}$ & $\begin{array}{c}0.280 \\
(0.016)\end{array}$ & $\begin{array}{c}0.023 \\
(0.020)\end{array}$ & $\begin{array}{c}0.188 \\
(0.010)\end{array}$ & $\begin{array}{c}0.865 \\
(0.008)\end{array}$ & $\begin{array}{c}0.000 \\
(0.006)\end{array}$ & $\begin{array}{c}0.026 \\
(0.001)\end{array}$ \\
\hline$\beta_{2}$ & $\pi_{1}$ & $\pi_{2}$ & BIC & & & \\
\hline $\begin{array}{c}0.942 \\
(0.007)\end{array}$ & $\begin{array}{c}0.500 \\
(0.008)\end{array}$ & 0.500 & 7153.053 & & & \\
\hline \multicolumn{7}{|c|}{ GJR-1component } \\
\hline$\mu_{1}$ & $\omega_{1}$ & $\alpha_{1}$ & $\lambda_{1}$ & $\beta_{1}$ & BIC & \\
\hline $\begin{array}{c}0.084 \\
(0.001)\end{array}$ & $\begin{array}{c}0.021 \\
(0.001)\end{array}$ & $\begin{array}{c}0.000 \\
(0.028)\end{array}$ & $\begin{array}{c}0.162 \\
(0.018)\end{array}$ & $\begin{array}{c}0.900 \\
(0.019)\end{array}$ & 7125.000 & \\
\hline \multicolumn{7}{|c|}{ AGARCH-2component } \\
\hline$\mu_{1}$ & $\mu_{2}$ & $\omega_{1}$ & $\alpha_{1}$ & $\lambda_{1}$ & $\beta_{1}$ & $\omega_{2}$ \\
\hline $\begin{array}{c}-0.042 \\
(0.146)\end{array}$ & $\begin{array}{c}0.272 \\
(0.254)\end{array}$ & $\begin{array}{c}0.000 \\
(0.059)\end{array}$ & $\begin{array}{c}0.160 \\
(0.156)\end{array}$ & $\begin{array}{c}0.795 \\
(0.114)\end{array}$ & $\begin{array}{c}0.822 \\
(0.118)\end{array}$ & $\begin{array}{c}0.000 \\
(0.018)\end{array}$ \\
\hline$\alpha_{2}$ & $\lambda_{2}$ & $\beta_{2}$ & $\pi_{1}$ & $\pi_{2}$ & BIC & \\
\hline $\begin{array}{c}0.018 \\
(0.060)\end{array}$ & $\begin{array}{c}0.312 \\
(0.476)\end{array}$ & $\begin{array}{c}0.957 \\
(0.120)\end{array}$ & $\begin{array}{c}0.656 \\
(0.579)\end{array}$ & 0.344 & 7076.656 & \\
\hline
\end{tabular}

Table 5 shows the best estimation results for Johnson \& Johnson in the three classes of GARCH $(1,1)$ families, i.e., GARCH $(1,1)$ model, AGARCH $(1,1)$ model, and GJR $(1,1)$ model. Standard errors, shown in parentheses, are obtained using asymptotic variance given in Equation (17) in Proposition 4. JNJ stands for Johnson \& Johnson. In models with more than one component, the probability of one component is determined automatically by the restriction of the sum of probabilities of all the components being equal to one so that its standard error is not given. 
Table 5. Estimation of the Three Classes of GARCH(1,1) Families in JNJ.

\begin{tabular}{cccccc}
\hline \multicolumn{6}{c}{ GARCH-2component } \\
\hline$\mu_{1}$ & $\mu_{2}$ & $\omega_{1}$ & $\alpha_{1}$ & $\beta_{1}$ & $\omega_{2}$ \\
\hline 0.075 & -0.010 & 0.020 & 0.101 & 0.771 & 0.052 \\
$(0.002)$ & $(0.009)$ & $(0.004)$ & $(0.004)$ & $(0.002)$ & $(0.005)$ \\
\hline$\alpha_{2}$ & $\beta_{2}$ & $\pi_{1}$ & $\pi_{2}$ & BIC \\
\hline 0.139 & 0.887 & 0.625 & 0.375 & 6073.109 \\
$(0.005)$ & $(0.012)$ & \multicolumn{5}{c}{$(0.049)$} \\
\hline \multicolumn{7}{c}{ GJR-1component } \\
\hline$\mu_{1}$ & $\omega_{1}$ & $\alpha_{1}$ & $\lambda_{1}$ & $\beta_{1}$ & BIC \\
\hline 0.048 & 0.039 & 0.023 & 0.161 & 0.846 & 6087.186 \\
$(0.000)$ & $(0.000)$ & $(0.000)$ & $(0.000)$ & $(0.000)$ \\
\hline \multicolumn{5}{c}{ AGARCH-1component } \\
\hline$\mu_{1}$ & $\omega_{1}$ & $\alpha_{1}$ & $\lambda_{1}$ & $\beta_{1}$ & BIC \\
\hline 0.048 & 0.004 & 0.091 & 0.675 & 0.850 & 6103.388 \\
$(0.000)$ & $(0.000)$ & $(0.000)$ & $(0.000)$ & $(0.000)$ \\
\hline \multicolumn{5}{c}{}
\end{tabular}

Table 6 shows the best estimation results for Amazon in the three classes of GARCH(1,1) families, i.e., GARCH $(1,1)$ model, AGARCH $(1,1)$ model, and GJR $(1,1)$ model. Standard errors, shown in parentheses, are obtained using asymptotic variance given in Equation (17) in Proposition 4. In models with more than one component, probability of one component is determined automatically by the restriction of the sum of probabilities of all the components being equal to one so that its standard error is not given.

Table 6. Estimation of the Three Classes of GARCH(1,1) Families in Amazon.

\begin{tabular}{|c|c|c|c|c|c|c|c|}
\hline \multicolumn{8}{|c|}{ GARCH-3component } \\
\hline$\mu_{1}$ & $\mu_{2}$ & $\mu_{3}$ & $\omega_{1}$ & $\alpha_{1}$ & $\beta_{1}$ & $\omega_{2}$ & $\alpha_{2}$ \\
\hline $\begin{array}{c}0.037 \\
(0.010)\end{array}$ & $\begin{array}{c}0.333 \\
(0.090)\end{array}$ & $\begin{array}{l}32.090 \\
(0.157)\end{array}$ & $\begin{array}{c}0.005 \\
(0.004)\end{array}$ & $\begin{array}{c}0.007 \\
(0.001)\end{array}$ & $\begin{array}{c}0.982 \\
(0.001)\end{array}$ & $\begin{array}{c}0.000 \\
(0.447)\end{array}$ & $\begin{array}{c}0.623 \\
(0.018)\end{array}$ \\
\hline$\beta_{2}$ & $\omega_{3}$ & $\alpha_{3}$ & $\beta_{3}$ & $\pi_{1}$ & $\pi_{2}$ & $\pi_{3}$ & BIC \\
\hline $\begin{array}{c}0.739 \\
(0.036)\end{array}$ & $\begin{array}{c}9.372 \\
(0.279)\end{array}$ & $\begin{array}{l}10.806 \\
(4.533)\end{array}$ & $\begin{array}{c}0.961 \\
(0.015)\end{array}$ & $\begin{array}{c}0.746 \\
(0.025)\end{array}$ & $\begin{array}{c}0.246 \\
(0.030)\end{array}$ & 0.008 & 10107.635 \\
\hline \multicolumn{8}{|c|}{ GJR-3component } \\
\hline$\mu_{1}$ & $\mu_{2}$ & $\mu_{3}$ & $\omega_{1}$ & $\alpha_{1}$ & $\lambda_{1}$ & $\beta_{1}$ & $\omega_{2}$ \\
\hline $\begin{array}{c}0.145 \\
(0.065)\end{array}$ & $\begin{array}{c}0.303 \\
(0.026)\end{array}$ & $\begin{array}{l}-0.375 \\
(0.085)\end{array}$ & $\begin{array}{c}0.022 \\
(0.010)\end{array}$ & $\begin{array}{c}0.006 \\
(0.001)\end{array}$ & $\begin{array}{c}0.045 \\
(0.017)\end{array}$ & $\begin{array}{c}0.969 \\
(0.003)\end{array}$ & $\begin{array}{c}0.000 \\
(0.000)\end{array}$ \\
\hline$\alpha_{2}$ & $\lambda_{2}$ & $\beta_{2}$ & $\omega_{3}$ & $\alpha_{3}$ & $\lambda_{3}$ & $\beta_{3}$ & $\pi_{1}$ \\
\hline $\begin{array}{c}0.000 \\
(0.000)\end{array}$ & $\begin{array}{c}0.000 \\
(0.001)\end{array}$ & $\begin{array}{c}0.999 \\
(0.002)\end{array}$ & $\begin{array}{c}0.000 \\
(0.024)\end{array}$ & $\begin{array}{c}0.003 \\
(0.002)\end{array}$ & $\begin{array}{c}0.591 \\
(0.051)\end{array}$ & $\begin{array}{c}0.758 \\
(0.010)\end{array}$ & $\begin{array}{c}0.460 \\
(0.086)\end{array}$ \\
\hline$\pi_{2}$ & $\pi_{3}$ & BIC & & & & & \\
\hline $\begin{array}{c}0.306 \\
(0.084)\end{array}$ & 0.234 & 10525.101 & & & & & \\
\hline \multicolumn{8}{|c|}{ AGARCH-3component } \\
\hline$\mu_{1}$ & $\mu_{2}$ & $\mu_{3}$ & $\omega_{1}$ & $\alpha_{1}$ & $\lambda_{1}$ & $\beta_{1}$ & $\omega_{2}$ \\
\hline $\begin{array}{c}0.270 \\
(0.447)\end{array}$ & $\begin{array}{l}-0.349 \\
(0.597)\end{array}$ & $\begin{array}{c}0.881 \\
(0.805)\end{array}$ & $\begin{array}{c}0.000 \\
(0.142)\end{array}$ & $\begin{array}{c}0.007 \\
(0.042)\end{array}$ & $\begin{array}{c}0.609 \\
(0.744)\end{array}$ & $\begin{array}{c}0.984 \\
(0.115)\end{array}$ & $\begin{array}{c}0.000 \\
(0.770)\end{array}$ \\
\hline$\alpha_{2}$ & $\lambda_{2}$ & $\beta_{2}$ & $\omega_{3}$ & $\alpha_{3}$ & $\lambda_{3}$ & $\beta_{3}$ & $\pi_{1}$ \\
\hline $\begin{array}{c}0.096 \\
(0.073)\end{array}$ & $\begin{array}{c}1.799 \\
(2.123)\end{array}$ & $\begin{array}{c}0.778 \\
(0.011)\end{array}$ & $\begin{array}{c}0.000 \\
(1.932)\end{array}$ & $\begin{array}{c}1.494 \\
(1.997)\end{array}$ & $\begin{array}{c}0.000 \\
(1.493)\end{array}$ & $\begin{array}{c}0.677 \\
(0.068)\end{array}$ & 0.524 \\
\hline$\pi_{2}$ & $\pi_{3}$ & BIC & & & & & \\
\hline $\begin{array}{c}0.360 \\
(0.714)\end{array}$ & $\begin{array}{c}0.117 \\
(0.158)\end{array}$ & 10204.766 & & & & & \\
\hline
\end{tabular}


Table 7 shows the best estimation results for Microsoft in the three classes of GARCH $(1,1)$ families, i.e., GARCH(1,1) model, AGARCH(1,1) model, and GJR(1,1) model. Standard errors, shown in parentheses, are obtained using asymptotic variance given in Equation (17) in Proposition 4. In models with more than one component, probability of one component is determined automatically by the restriction of the sum of probabilities of all the components being equal to one so that its standard error is not given.

Table 7. Estimation of the Three Classes of GARCH(1,1) Families in Microsoft.

\begin{tabular}{|c|c|c|c|c|c|c|c|}
\hline \multicolumn{8}{|c|}{ GARCH-3component } \\
\hline$\mu_{1}$ & $\mu_{2}$ & $\mu_{3}$ & $\omega_{1}$ & $\alpha_{1}$ & $\beta_{1}$ & $\omega_{2}$ & $\alpha_{2}$ \\
\hline $\begin{array}{c}-0.016 \\
(0.000)\end{array}$ & $\begin{array}{c}0.331 \\
(0.000)\end{array}$ & $\begin{array}{l}14.043 \\
(0.006)\end{array}$ & $\begin{array}{c}0.001 \\
(0.000)\end{array}$ & $\begin{array}{c}0.028 \\
(0.000)\end{array}$ & $\begin{array}{c}0.948 \\
(0.000)\end{array}$ & $\begin{array}{c}2.571 \\
(0.005)\end{array}$ & $\begin{array}{c}0.622 \\
(0.001)\end{array}$ \\
\hline$\beta_{2}$ & $\omega_{3}$ & $\alpha_{3}$ & $\beta_{3}$ & $\pi_{1}$ & $\pi_{2}$ & $\pi_{3}$ & BIC \\
\hline $\begin{array}{c}0.067 \\
(0.000)\end{array}$ & $\begin{array}{c}3.539 \\
(0.063)\end{array}$ & $\begin{array}{c}1.522 \\
(0.022)\end{array}$ & $\begin{array}{c}0.985 \\
(0.000)\end{array}$ & $\begin{array}{c}0.751 \\
(0.000)\end{array}$ & $\begin{array}{c}0.239 \\
(0.000)\end{array}$ & 0.010 & 8611.326 \\
\hline \multicolumn{8}{|c|}{ GJR-3component } \\
\hline$\mu_{1}$ & $\mu_{2}$ & $\mu_{3}$ & $\omega_{1}$ & $\alpha_{1}$ & $\lambda_{1}$ & $\beta_{1}$ & $\omega_{2}$ \\
\hline $\begin{array}{c}0.156 \\
(0.058)\end{array}$ & $\begin{array}{l}-0.159 \\
(0.045)\end{array}$ & $\begin{array}{l}-0.043 \\
(0.178)\end{array}$ & $\begin{array}{c}0.028 \\
(0.016)\end{array}$ & $\begin{array}{c}0.029 \\
(0.005)\end{array}$ & $\begin{array}{c}0.000 \\
(0.021)\end{array}$ & $\begin{array}{c}0.954 \\
(0.007)\end{array}$ & $\begin{array}{c}0.000 \\
(0.049)\end{array}$ \\
\hline$\alpha_{2}$ & $\lambda_{2}$ & $\beta_{2}$ & $\omega_{3}$ & $\alpha_{3}$ & $\lambda_{3}$ & $\beta_{3}$ & $\pi_{1}$ \\
\hline $\begin{array}{c}0.005 \\
(0.005)\end{array}$ & $\begin{array}{c}0.641 \\
(0.059)\end{array}$ & $\begin{array}{c}0.783 \\
(0.038)\end{array}$ & $\begin{array}{c}0.000 \\
(0.003)\end{array}$ & $\begin{array}{c}0.000 \\
(0.000)\end{array}$ & $\begin{array}{c}0.000 \\
(0.004)\end{array}$ & $\begin{array}{c}0.998 \\
(0.003)\end{array}$ & $\begin{array}{c}0.569 \\
(0.028)\end{array}$ \\
\hline$\pi_{2}$ & $\pi_{3}$ & BIC & & & & & \\
\hline 0.253 & $\begin{array}{c}0.177 \\
(0.061)\end{array}$ & 8835.438 & & & & & \\
\hline \multicolumn{8}{|c|}{ AGARCH-3component } \\
\hline$\mu_{1}$ & $\mu_{2}$ & $\mu_{3}$ & $\omega_{1}$ & $\alpha_{1}$ & $\lambda_{1}$ & $\beta_{1}$ & $\omega_{2}$ \\
\hline $\begin{array}{c}0.055 \\
(0.047)\end{array}$ & $\begin{array}{c}0.209 \\
(0.188)\end{array}$ & $\begin{array}{l}-0.185 \\
(0.607)\end{array}$ & $\begin{array}{c}0.000 \\
(0.058)\end{array}$ & $\begin{array}{c}0.027 \\
(0.027)\end{array}$ & $\begin{array}{c}0.885 \\
(0.547)\end{array}$ & $\begin{array}{c}0.949 \\
(0.037)\end{array}$ & $\begin{array}{c}0.000 \\
(0.076)\end{array}$ \\
\hline$\alpha_{2}$ & $\lambda_{2}$ & $\beta_{2}$ & $\omega_{3}$ & $\alpha_{3}$ & $\lambda_{3}$ & $\beta_{3}$ & $\pi_{1}$ \\
\hline $\begin{array}{c}1.087 \\
(2.615) \\
\end{array}$ & $\begin{array}{c}0.591 \\
(0.563) \\
\end{array}$ & $\begin{array}{c}0.637 \\
(0.376) \\
\end{array}$ & $\begin{array}{c}0.000 \\
(0.784) \\
\end{array}$ & $\begin{array}{c}0.132 \\
(0.095) \\
\end{array}$ & $\begin{array}{c}1.783 \\
(1.765) \\
\end{array}$ & $\begin{array}{c}0.018 \\
(0.463) \\
\end{array}$ & $\begin{array}{c}0.751 \\
(0.148) \\
\end{array}$ \\
\hline$\pi_{2}$ & $\pi_{3}$ & BIC & & & & & \\
\hline 0.134 & $\begin{array}{c}0.114 \\
(0.069)\end{array}$ & 8656.691 & & & & & \\
\hline
\end{tabular}

The best model in the individual stocks of Johnson \& Johnson, Amazon, and Microsoft are GARCH(1,1) two and three components models. The best model in Johnson \& Johnson is a GARCH(1,1) two components model where the dominant state is with positive and significant mean and another state is a negative shock state with negative mean. The estimate of $\alpha$, the effect of previous innovation, is larger in the negative shock state than in the dominant state, which is usually the case in previous studies of this kind (cf., e.g., Alexander and Lazar 2009). However, the estimate of $\beta$, the effect of previous conditional variance, is larger in the negative shock state than in the dominant state with positive mean, which differs from many of previous studies of this kind (cf., e.g., Alexander and Lazar 2009). The best model in Amazon is a $\operatorname{GARCH}(1,1)$ three components model with distinctive parameter estimates. It has three components with positive and significant mean in every element. In particular, the second and third components are both positive shock states with the third one being extraordinary size. The estimate of $\alpha$ is also huge in the extraordinary third component and large in the second component while the estimate of $\beta$ is not small in these components. The best model in Microsoft is also a GARCH $(1,1)$ three components model. The second and third components are both positive shock states with positive and significant mean. The third component is also a positive shock state of very large size. The estimate of $\alpha$ is large in the second component and very large in the third component, indicating the impact of previous innovation is large in these components. 
The estimate of $\beta$ in the extraordinary third component is not different from that in the dominant state of the first component while that in the second positive shock state is less than those in the first and third components.

We also provide diagnostic test results for the best model in each stock with respect to the BIC in Table 8 . We describe the diagnostic test results in Table 8 only for the best model in each stock below. The diagnostic test results show no serial correlation up to the twelfth-order in the squared normalized residuals, no significance of the sign bias test except Johnson \& Johnson, significance of the negative size bias test except NASDAQ, and significance of the negative effect of the positive size bias test in each stock which indicates the effect of previous positive residuals is negative, which is different from the result of Engle and $\mathrm{Ng}$ (1993) for the Japanese stock index. The joint test for the three biases is highly significant for all the stocks. The results of the sign bias test, negative size bias test, positive sign bias test, and joint test are those applied to the normalized residuals.

Table 8. Diagnostic Test Results.

\begin{tabular}{ccccccc}
\hline Name & Model & LB (12) & Sign Bias & - Size Bias & + Size Bias & Joint \\
\hline \multirow{3}{*}{ DOW } & GARCH-2 & 12.207 & $2.937^{* *}$ & $-7.248^{* *}$ & $-6.915^{* *}$ & $53.312^{* *}$ \\
& GJR-1 & 16.524 & $2.142^{*}$ & $-3.017^{* *}$ & $-5.651^{* *}$ & $19.328^{* *}$ \\
& AGARCH-2 & 15.988 & 1.698 & $-2.936^{* *}$ & $-3.430^{* *}$ & $18.028^{* *}$ \\
\hline \multirow{2}{*}{ NASDAQ } & GARCH-2 & 7.582 & $3.054^{* *}$ & $-5.623^{* *}$ & $-7.323^{* *}$ & $45.817^{* *}$ \\
& GJR-1 & 10.457 & 1.723 & 0.527 & $-5.631^{* *}$ & $13.937^{* *}$ \\
& AGARCH-2 & 11.256 & 1.101 & -1.165 & $-4.339^{* *}$ & $14.438^{* * *}$ \\
\hline \multirow{3}{*}{ JNJ } & GARCH-2 & 14.406 & $2.170^{*}$ & $-7.164^{* *}$ & $-5.764^{* *}$ & $48.664^{* *}$ \\
& GJR-1 & 13.615 & 1.830 & $-9.847^{* *}$ & $-6.345^{* *}$ & $39.013^{* *}$ \\
& AGARCH-1 & 14.303 & 1.672 & $-6.182^{* *}$ & $-3.967^{* *}$ & $28.309^{* *}$ \\
\hline \multirow{3}{*}{ Amazon } & GARCH-3 & 10.518 & -0.612 & $-2.299^{* *}$ & $-2.487^{*}$ & $14.173^{* *}$ \\
& GJR-3 & 20.986 & -1.461 & 0.309 & -0.191 & 5.718 \\
& AGARCH-3 & 12.277 & $-2.990^{* *}$ & -1.469 & -1.500 & 7.800 \\
\hline \multirow{2}{*}{ Microsoft } & GARCH-3 & 5.075 & 1.230 & $-3.303^{* *}$ & $-2.330^{*}$ & $11.034^{*}$ \\
& GJR-3 & 7.123 & 1.075 & $-7.751^{* *}$ & -0.302 & $13.327^{* *}$ \\
& AGARCH-3 & 7.158 & 1.504 & $-2.445^{*}$ & -0.871 & 6.206 \\
\hline
\end{tabular}

JNJ stands for Johnson \& Johnson. One and two asterisks of * and ** indicate significance at the 5 and 1 percent levels respectively.

Table 8 provides diagnostic test results for the best model in the three classes of GARCH $(1,1)$ families with respect to the BIC in the stock return data. LB (12) denotes Ljung-Box statisics, modified by Diebold (1988), of serial correlation of the twelfth-order serial correlation in the squared normalized residuals. Sign Bias, - Size Bias, + Size Bias, and Joint denote respectively the sign bias test statistic, negative size bias test statistic, positive sign bias test statistic, and joint test statistic. These tests are applied to the normalized residuals, i.e., residuals divided by the conditional standard deviation estimate. In the table, GARCH-2,GJR-1, and AGARCH-2 in the DOW denote respectively a GARCH(1,1) two components model, a GJR $(1,1)$ one component model, and an $\mathrm{AGARCH}(1,1)$ two components model. Other names of these models in the table are similarly defined.

We provide summary statistics of the conditional variance estimate of the best model with respect to the BIC in the three classes of GARCH $(1,1)$ families in each stock in Table 9. The variation of the estimated conditional variance of the best model is a lot smaller than that of return data, which was also true in Engle and $\mathrm{Ng}$ (1993). We remark that mean of the estimated conditional variance of the best model for Amazon, i.e., a GARCH $(1,1)$ three components model, is five times as large as that of return data, which is exceptional in the conditional variance estimate of the best model. 
Table 9. Summary Statistics of the Conditional Variance Estimate.

\begin{tabular}{cccccccc}
\hline Name & Model & mean & s.d. & min & max & Skewness & Kurtosis \\
\hline \multirow{2}{*}{ DOW } & $\epsilon^{2}$ & 1.491 & 5.344 & 0.000 & 122.774 & 12.860 & 239.113 \\
& GARCH-2 & 1.565 & 2.960 & 0.155 & 31.202 & 5.478 & 38.808 \\
& GJR-1 & 1.385 & 2.534 & 0.155 & 25.469 & 4.952 & 31.874 \\
& AGARCH-2 & 1.399 & 2.416 & 0.154 & 25.829 & 5.393 & 38.338 \\
\hline NASDAQ & $\epsilon^{2}$ & 1.982 & 6.088 & 0.000 & 139.380 & 10.599 & 172.490 \\
& GARCH-2 & 1.970 & 3.051 & 0.278 & 29.339 & 4.569 & 28.106 \\
& GJR-1 & 1.826 & 2.938 & 0.253 & 27.901 & 4.553 & 27.263 \\
& AGARCH-2 & 1.835 & 2.613 & 0.225 & 28.340 & 4.631 & 29.988 \\
\hline \multirow{2}{*}{ JNJ } & $\epsilon^{2}$ & 1.114 & 4.406 & 0.000 & 149.554 & 20.575 & 602.706 \\
& GARCH-2 & 1.046 & 1.603 & 0.266 & 24.774 & 7.527 & 75.450 \\
& GJR-1 & 0.942 & 1.334 & 0.282 & 16.558 & 6.184 & 50.521 \\
& AGARCH-1 & 0.942 & 1.391 & 0.154 & 20.176 & 7.028 & 66.419 \\
\hline \multirow{2}{*}{ Amazon } & $\epsilon^{2}$ & 6.192 & 22.819 & 0.000 & 717.976 & 16.600 & 436.382 \\
& GARCH-3 & 30.580 & 20.555 & 12.878 & 200.709 & 3.238 & 15.634 \\
& GJR-3 & 4.770 & 4.992 & 0.852 & 58.587 & 4.105 & 26.815 \\
& AGARCH-3 & 6.410 & 8.290 & 1.019 & 151.917 & 6.106 & 68.334 \\
\hline \multirow{2}{*}{ Microsoft } & $\epsilon^{2}$ & 3.217 & 11.519 & 0.000 & 346.133 & 15.471 & 374.684 \\
& GARCH-3 & 10.154 & 5.588 & 5.070 & 80.412 & 3.832 & 24.191 \\
& GJR-3 & 2.785 & 3.327 & 0.510 & 35.401 & 3.998 & 22.861 \\
& AGARCH-3 & 3.114 & 4.142 & 0.501 & 67.928 & 5.768 & 55.125 \\
\hline
\end{tabular}

JNJ stands for Johnson \& Johnson.

Table 9 provides summary statistics of the conditional variance estimate of the best model of the three classes of GARCH $(1,1)$ families in each stock with respect to the BIC. In the table, $\epsilon^{2}$ denotes the squared stock return. In the table, s.d., min, and max stand for respectively standard deviation, a minimum value, and a maximum value. In the table, GARCH-2, GJR-1, and AGARCH-2 in the DOW denote respectively a GARCH $(1,1)$ two components model, a GJR $(1,1)$ one component model, and an AGARCH $(1,1)$ two components model. Other names of these models in the table are similarly defined.

Before we show the AS performance index for multi-period gambles, we first show the Sharpe ratio obtained from return data and the AS performance index for one-period gambles. The Sharpe ratio scores are the same as those in Hodoshima (2019) who estimated the same data. This is reproduced in Table 10. The Sharpe ratio is larger in NASDAQ than in DOW. The highest Sharpe ratio score 0.051 is for Amazon while the second highest one 0.041 is for Johnson \& Johnson. Hodoshima (2019) estimated a performance index named inner rate of risk aversion (IRRA) proposed by Miyahara $(2010,2014)$, which is equivalent to the AS performance index for one-period gambles (see also Hodoshima and Miyahara, 2020 for the relationship between utility indifference pricing and the AS performance index for one-period gambles when the investor is risk averse as well as risk loving), assuming the underlying stochastic process of return data follows the normal mixture process with time-invariant volatility. Hodoshima (2019) estimated the AS performance index for one-period gambles by the MLE using the E-M algorithm. We reproduce the estimate of the AS performance index for one-period gambles in Table 11. We remark that the MLE of the AS performance index for one-period gambles in the parametric normal mixture model with time-invariant volatility is little different from the nonparametric generalized method of moments (GMM) estimate, which was employed in Kadan and Liu (2014). The estimates of the AS performance index for one-period gambles in the two indexes, DOW and NASDAQ, are both larger than the Sharpe ratio scores. That is also true in other individual stocks of Johnson \& Johnson and Microsoft. On the other hand, Amazon is a stock with its AS performance index less than the Sharpe ratio. Johnson \& Johnson and Amazon are two contrasting stocks in Hodoshima (2019). Johnson \& Johnson is rated fairly well by the Sharpe ratio but rated the best by the AS performance index. On the other hand, Amazon is rated the best by the Sharpe ratio but rated mediocre by the AS performance index. This is because Amazon is the stock with the highest 
mean and highest risk, which causes it to take negative values often, which makes its AS performance score low. On the other hand, stable stocks such as Johnson \& Johnson perform fairly well, which are rated highly by the AS performance index (cf. Hodoshima 2019).

Table 10. The Sharpe Ratio in Ascending Order for the Stock Returns.

\begin{tabular}{cc}
\hline Name & Sharpe Ratio \\
\hline DOW & 0.021 \\
Microsoft & 0.024 \\
NASDAQ & 0.031 \\
JNJ & 0.041 \\
Amazon & 0.051 \\
\hline \multicolumn{2}{c}{ JNJ stands for Johnson \& Johnson. }
\end{tabular}

Table 11. The AS Performance Index for One-period Gambles in Ascending Order for the Stock Returns. Table 11 presents the AS performance index for one-period gambles assuming the underlying stochastic process to be a normal mixture process with time-invariant volatility.

\begin{tabular}{cc}
\hline Name & AS Index \\
\hline Microsoft & 0.027 \\
DOW & 0.037 \\
Amazon & 0.042 \\
NASDAQ & 0.046 \\
JNJ & 0.079 \\
\hline
\end{tabular}

JNJ stands for Johnson \& Johnson.

To compare the fitness of the best model in the models for one-period gambles and multi-period gambles, we provide scores of the BIC in the best model of the normal mixture process with time-invariant volatility in Table 12. When we compare scores of the best BIC in the models for one-period gambles with those for multi-period gambles, given in Tables 3-7, we can see the best models for multi-period gambles dominate those for one-period gambles. Hence, the model selection criterion of the BIC uniformly chooses the model for multi-period gambles as compared to the model for one-period gambles in all the stocks we consider.

Table 12. The BIC of the Best Model of One-period Gambles for the Stock Returns.

\begin{tabular}{cc}
\hline Name & BIC \\
\hline DOW & 6822.029 \\
NASDAQ & 7667.211 \\
JNJ & 6379.585 \\
Amazon & 10308.100 \\
Microsoft & 8805.842 \\
\hline \multicolumn{2}{c}{ JNJ stands for Johnson \& Johnson. }
\end{tabular}

We then show the AS performance index for multi-period gambles in Table 13. We set the discount factor $\rho$ to be $0.01,0.05,0.10$ in annual rate. We show the AS performance index for the best model by the BIC as well as the second best model by the BIC to see how the result changes as the model changes in all the stocks we consider. The results show the AS performance index for multi-period gambles tends to be larger than that for one-period gambles when the AS performance index for one-period gambles is larger than the Sharpe ratio. The AS performance index for one-period gambles is larger than the Sharpe ratio in four stocks out of the five stocks we examine in this paper, i.e., DOW, NASDAQ, Johnson \& Johnson, and Microsoft. One exception of the above property, i.e., the AS performance index for multi-period gambles tends to be larger than that for one-period gambles when the AS performance index for one-period gambles is larger than the Sharpe ratio, is Johnson \& Johnson 
where the AS performance index for multi-period gambles is similar to that for one-period gambles in the best model of the GARCH two components model. However, the AS performance index for multi-period gambles is distinctively larger than that for one-period gambles in the second-best model of the GJR one component model in Johnson \& Johnson. We remark the same is true in the third-best model of the AGARCH one component model in Johnson \& Johnson where the AS performance index for multi-period gambles is $0.101,0.098$, and 0.093 respectively when $\rho=0.01,0.05$, and 0.10 . The AS performance index for one-period gambles is smaller than the Shape ratio only in Amazon among the five stocks we examine in this paper. The AS performance index for multi-period gambles is even smaller than that for one-period gambles when the AS performance index for one-period gambles is smaller than the Sharpe ratio. This occurs in the case of Amazon. When we compare the AS performance index with the Sharpe ratio, a favorable evaluation score tends to become even better in multi-period gambles than in one-period gambles while an unfavorable evaluation score tends to become even worse in multi-period gambles than in one-period gambles. This property is new, although our results are limited since we only examine a handful of stocks. Whether this property is only empirical or an intrinsic property of the relationship between the AS performance index and Sharpe ratio is beyond the scope of the present study and is left as a future research topic to be studied.

Table 13. The AS Performance Index for Multi-Period Gambles for the Stock Returns.

\begin{tabular}{cccccc}
\hline Name & & & $\boldsymbol{\rho}=\mathbf{0 . 0 1}$ & $\boldsymbol{\rho}=\mathbf{0 . 0 5}$ & $\boldsymbol{\rho} \mathbf{0 . 1}$ \\
\hline \multirow{2}{*}{ DOW } & MinBIC & AGARCH-2 & 0.051 & 0.048 & 0.044 \\
& 2ndMinBIC & GJR-1 & 0.068 & 0.063 & 0.058 \\
\hline \multirow{2}{*}{ NASDAQ } & MinBIC & AGARCH-2 & 0.070 & 0.066 & 0.062 \\
& 2ndMinBIC & GJR-1 & 0.089 & 0.084 & 0.078 \\
\hline \multirow{2}{*}{ JNJ } & MinBIC & GARCH-2 & 0.080 & 0.078 & 0.074 \\
& 2ndMinBIC & GJR-1 & 0.100 & 0.096 & 0.092 \\
\hline \multirow{2}{*}{ Amazon } & MinBIC & GARCH-3 & 0.025 & 0.025 & 0.024 \\
& 2ndMinBIC & AGARCH-3 & 0.036 & 0.034 & 0.032 \\
\hline \multirow{2}{*}{ Microsof } & MinBIC & GARCH-3 & 0.047 & 0.046 & 0.044 \\
& 2ndMinBIC & AGARCH-3 & 0.031 & 0.029 & 0.028 \\
\hline
\end{tabular}

JNJ stands for Johnson \& Johnson.

Table 13 presents the AS performance index for multi-period gambles in the best model and second best model with respect to the BIC of the three classes of GARCH $(1,1)$ families. MinBIC and 2ndMinBIC stand for respectively the best model and second best model with respect to the BIC. In the table, AGARCH-2 and GJR-1 in the DOW denote respectively an AGARCH(1,1) two components model and a GJR $(1,1)$ one component model. Other names of these models in the table are similarly defined.

\section{Concluding Comments}

In this paper, we presented an empirical study of the AS performance index for multi-period gambles under the setup given by Kadan and Liu (2014) where the utility function of multi-period gambles is time-separable with a discount factor. We obtained the parametric estimates of the AS performance index for multi-period gambles assuming the underlying stochastic process of returns to follow the normal mixture process with time-varying volatility of $\mathrm{GARCH}$ families. Our empirical study of the AS performance index is the first empirical study of the AS performance index for multi-period gambles. We compared the AS performance index for multi-period gambles with that for one-period gambles as well as the Sharpe ratio obtained from data. Estimates of the AS performance index for one-period gambles are obtained assuming the underlying stochastic process of returns to follow the normal mixture process with time-invariant volatility. 
Our results show the following property of the estimates of the AS performance index for multi-period gambles. Stocks with higher AS performance index scores for one-period gambles than the Sharpe ratio are evaluated even better in the AS performance index for multi-period gambles. On the other hand, stocks with lower AS performance index scores for one-period gambles than the Sharpe ratio are evaluated even worse in the AS performance index for multi-period gambles. Our results are obtained using a selection of U.S. stocks and show obtaining the AS performance index for multi-period gambles is not difficult under a reasonable and tractable assumption of the normal mixture process with time-varying volatility of GARCH families. Therefore, the AS performance index for multi-period gambles is a practical tool for evaluation of multi-period gambles, which opens up a new way of evaluating various dynamic gambles. Obtaining appropriate performance measures is essential to investors, financial managers, and regulators, we believe our approach of the AS performance index for multi-period gambles is widely useful to these audiences. Exploring the AS performance index for multi-period gambles with the different class of assets, projects, cash flows, etc. is important and interesting and left as a future research topic.

Author Contributions: Conceptualization, J.H. and T.Y.; Methodology, J.H. and T.Y.; Software, J.H. and T.Y.; Validation, J.H. and T.Y.; Formal Analysis, J.H.; Investigation, J.H. and T.Y.; Resources, J.H. and T.Y.; Data Curation, T.Y.; Writing-Original Draft Preparation, J.H.; Writing—Review \& Editing, J.H. and T.Y.; Visualization, J.H. and T.Y.; Supervision, J.H.; Project Administration, J.H.; Funding Acquisition, J.H. All authors have read and agreed to the published version of the manuscript.

Funding: This work was supported by JSPS KAKENHI Grant Number JP17K03667.

Conflicts of Interest: The authors declare no conflict of interest.

\section{References}

Alexander, Carol. 2004. Normal mixture diffusion with uncertain volatility: Modelling short- and long-term smile effects. Journal of Banking and Finance 28: 2957-80. [CrossRef]

Alexander, Carol, and Emese Lazar. 2009. Modelling regime-specific stock price volatility. Oxford Bulletin of Economics and Statistics 71: 761-97. [CrossRef]

Aumann, Robert J., and Roberto Serrano. 2008. An economic index of riskiness. Journal of Political Economy 116: 810-36. [CrossRef]

Bollerslev, Tim. 1986. Generalized autoregressive conditional heteroskedasticity. Journal of Econometrics 31: 307-27. [CrossRef]

Cherny, Alexander, and Dilip Madan. 2009. New measures for performance evaluation. Review of Financial Studies 22: 2571-606. [CrossRef]

Cogneau, Philippe, and Georges Hubner. 2009a. The (more than) 100 ways to measure portfolio performance. Part 1: Standardized risk-adjusted measure. Journal of Performance Measurement 13: 56-71.

Cogneau, Philippe, and Georges Hubner. 2009b. The (more than) 100 ways to measure portfolio performance. Part 2: Special measures and comparison. Journal of Performance Measurement 14: 56-69.

Diebold, Francis X. 1988. Empirical Modeling of Exchange Rate Dynamics. Berlin and Heidelberg: Springer.

Engle, Robert F. 1982. Autoregressive conditional heteroscedasticity with estimates of the variance of United Kingdom inflation. Econometrica 50: 987-1007. [CrossRef]

Engle, Robert F. 1990. Stock volatility and the crash of '87: Discussion. The Review of Financial Studies 3: $103-6$. [CrossRef]

Engle, Robert F., and Victor K. Ng. 1993. Measuring and testing the impact of news on volatility. Journal of Finance 48: 1749-78. [CrossRef]

Everitt, Brian S., and D. J. Hand. 1981. Finite Mixture Distributions. New York: Chapman and Hall.

Foster, Dean P., and Sergiu Hart. 2009. An operational measure of riskiness. Journal of Political Economy 117: 785-814. [CrossRef]

Glosten, Lawrence R., Ravi Jagannathan, and David E. Runkle. 1993. On the relationship between expected value and the volatility of excess returns on stocks. Journal of Finance 48: 1779-1801. [CrossRef] 
Goetzmann, William, Jonathan Ingersoll, Matthew Spiegel, and Ivo Welch. 2007. Portfolio performance manipulation and manipulation-proof performance measures. Review of Financial Studies 20: 1503-46. [CrossRef]

Haas, Markus, Stefan Mittnik, and Marc S. Paolella. 2004. Mixed normal conditional heteroskedasticity. Journal of Financial Econometrics 2: 211-50. [CrossRef]

Hart, Sergiu. 2011. Comparing risks by acceptance and rejection. Journal of Political Economy 119: 617-38. [CrossRef]

Heathcote, Christopher R. 1977. Integrated mean square error estimation of parameters. Biometrika 64: $255-64$. [CrossRef]

Hodoshima, Jiro. 2019. Stock performance by utility indifference pricing and the Sharpe ratio. Quantitative Finance 19: 327-38. [CrossRef]

Hodoshima, Jiro. 2020. On the appropriate performance measure based on the Aumann-Serrano index. Paper presented at JAFEE 2020 Summer Meetings, online conference, 29 August 2020.

Hodoshima, Jiro, and Nana Otsuki. 2019. Evaluation by the Aumann and Serrano performance index and Sharpe ratio: Bitcoin performance. Applied Economics 51: 4282-98. [CrossRef]

Hodoshima, Jiro, and Yoshio Miyahara. 2020. Utility indifference pricing and the Aumann-Serrano performance index. Journal of Mathematical Economics 86: 83-89. [CrossRef]

Homm, Ulrich, and Christian Pigorsch. 2012a. Beyond the Sharpe ratio: An application of the Aumann-Serrano index to performance measurement. Journal of Banking $\mathcal{E}$ Finance 36: 2274-84.

Homm, Ulrich, and Christian Pigorsch. 2012b. An operational interpretation and existence of the Aumann-Serrano index of riskiness. Economics Letters 114: 265-67. [CrossRef]

Kadan, Ohad, and Fang Liu. 2014. Performance evaluation with high moments and disaster risk. Journal of Financial Economics 113: 131-55. [CrossRef]

Kon, Stanley J. 1984. Models of stock returns-a comparison. Journal of Finance 39: 147-65.

McLachlan, Geoffrey J., and David Peel. 2000. Finite Mixture Models: Inference and Applications to Clustering. New York: Marcel Dekker.

Miyahara, Yoshio. 2010. Risk-sensitive value measure method for projects evaluation. Journal of Real Options and Strategy 3: 185-204. [CrossRef]

Miyahara, Yoshio. 2014. Evaluation of the scale risk. RIMS Kokyuroku 1886: 181-88.

Niu, Cuizhen, Xu Guo, Michael McAleer, and Wing-Keung Wong. 2018. Theory and application of an economic performance measure. International Review of Economics and Finance 56: 383-96. [CrossRef]

Sculze, Klaas. 2014. Existence and computation of the Aumann-Serrano index of riskiness. Journal of Mathematical Economics 50: 219-24. [CrossRef]

Titterington, D. Michael, Adrian F. M. Smith, and Udi E. Makov. 1985. Statistical Analysis of Finite Mixture Distributions. New York: Wiley.

$\mathrm{Xu}$, Dinghai, and Tony S. Wirjanto. 2010. An empirical characteristic function approach to VaR under a mixure-of-normal distribution with time-varying volatility. The Journal of Derivatives 18: 39-58. [CrossRef]

Publisher's Note: MDPI stays neutral with regard to jurisdictional claims in published maps and institutional affiliations.

(C) 2020 by the authors. Licensee MDPI, Basel, Switzerland. This article is an open access article distributed under the terms and conditions of the Creative Commons Attribution (CC BY) license (http://creativecommons.org/licenses/by/4.0/). 